\title{
ON BARY-STECHKIN THEOREM
}

\section{A.I. RUBINSHTEIN}

Dedicated to professor Sergey Alexandrovich Telyakovsky to his 85th anniversary

\begin{abstract}
In the beginning of the past century, N.N. Luzin proved almost everywhere convergence of an improper integral representing the function $\bar{f}$ conjugated to a $2 \pi$-periodic summable with a square function $f(x)$. A few years later I.I. Privalov proved a similar fact for a summable function. V.I. Smirnov showed that if $\bar{f}$ is summable, then its Fourier series is conjugate to the Fourier series for $f(x)$. It is easy to see that if $f(x) \in \operatorname{Lip} \alpha, 0<\alpha<1$, then $\bar{f}(x) \in \operatorname{Lip} \alpha$. The Hilbert transformation for $f(x)$ differs from $\bar{f}(x)$ by a bounded function and has a simpler kernel. It is easy to show that the Hilbert transformation of $f(x) \in \operatorname{Lip} \alpha, 0<\alpha<1$, also belongs to $\operatorname{Lip} \alpha$. In 1956 N.K. Bari and S.B. Stechkin found the necessary and sufficient condition on the modulus of continuity $f(x)$ for the function $\bar{f}(x)$ to have the same modulus of continuity. In 2016, the author introduced the concept of conjugate function as Hilbert transformation for functions defined on a dyadic group. In the present paper we show an analogue of the Bari-Stechkin (and Privalov) theorem fails that for a conjugated in this sense function.
\end{abstract}

Keywords: dyadic group, conjugate function, modulus of continuity, Bari-Stechkin theorem.

\section{Mathematics Subject Classification: 42A50}

As it is known, see, for instance, [1, Ch. VIII], the integral operator

$$
(\Phi f)(x)=\lim _{\varepsilon \rightarrow+0}-\frac{1}{\pi} \int_{\varepsilon}^{\pi} \frac{f(x+t)-f(x-t)}{2 \tan t / 2} d t
$$

maps each $2 \pi$-periodic function $f(x) \in L(-\pi ; \pi)$ into a function $\widetilde{f}(x)$ called conjugate to $f(x)$ and being the imaginary part of the power series on the circumference $|z|=1$. Integral (1) exists almost everywhere on $[-\pi ; \pi]$ for all $f(x) \in L_{2}(-\pi ; \pi)$ [2] and for $f(x) \in L(-\pi ; \pi)$ [3].

In [3], I.I. Privalov showed that if $|f(x+h)-f(x)| \leqslant C \cdot|h|^{\alpha}, 0<\alpha<1$, i.e., $f(x) \in \operatorname{Lip} \alpha$, $0<\alpha<1$, then

$$
\widetilde{f}(x)=-\frac{1}{\pi} \int_{0}^{\pi} \frac{f(x+t)-f(x-t)}{2 \tan \frac{t}{2}} d t
$$

everywhere and $\widetilde{f}(x)$ also belongs to $\operatorname{Lip} \alpha$.

In [4], N.K. Bari showed that if a continuous monotonically increasing function $\varphi(\delta)$ obeys the conditions: there exists a constant $C>1$ such that

$$
1<\varliminf_{\delta \rightarrow 0} \frac{\varphi(C \delta)}{\varphi(\delta)} \leqslant \varlimsup_{\delta \rightarrow 0} \frac{\varphi(C \delta)}{\varphi(\delta)}<C,
$$

A.I. Rubinshtein, On BARY-Stechkin theorem.

(c) Rubinshtein A.I. 2019.

Submitted August 18, 201\%. 
then it follows from the condition

$$
\omega(\delta, f)=\sup _{|h| \leqslant \delta, x \in[-\pi ; \pi]}|f(x+h)-f(x)|=O(\varphi(\delta))
$$

that

$$
\omega(\delta, \widetilde{f})=O(\varphi(\delta)) .
$$

In [5], N.K. Bari and S.B. Stechkin showed that if in addition to the monotonicity of $\varphi(\delta)$ we also suppose that $\frac{\varphi(\delta)}{\delta}$ is non-increasing, then 3 is also a necessary condition for 5 . That is, there was established criterion (3) for the functions $f(x)$ and $\widetilde{f}(x)$ to belong to the same class, i.e., the have the same smoothness.

It was mentioned many times (first by N.N. Luzin), that instead of the functions $\widetilde{f}(x),(1)$, (2), it is more convenient to consider the function

$$
F(x)=\int_{0}^{\pi} \frac{f(x+t)-f(x-t)}{t} d t
$$

where the integral treated in the sense of the Cauchy principal value.

By almost literal reproducing of arguing from [1], one can confirm that if $f(x) \in \operatorname{Lip} \alpha$, $0<\alpha<1$, then the function $F(x)$ in (6) belongs to $\operatorname{Lip} \alpha, 0<\alpha<1$.

Let $G$ be a set of sequence formed by 0 and 1 , in which we introduce the operation "+ + " of coordinate-wise addition modulo 2 , that is,

$$
\left\{\begin{array}{l}
G=\left\{x=\left(x_{1}, x_{2}, \ldots\right), x_{k} \in\{0 ; 1\}\right\} \\
x \dot{+} y=\left(x_{1}, x_{2}, \ldots\right) \dot{+}\left(y_{1}, y_{2}, \ldots\right)=z=\left(z_{1}, z_{2}, \ldots\right), \text { where } z_{k}=\left(x_{k}+y_{k}\right)(\bmod 2) .
\end{array}\right.
$$

In view of 77 it is obvious that $G$ is an Abelian (commutative) group and becomes a topological commutative group if we define the topology by means of the system of neighbourhoods of the zero element $O=(0,0, \ldots)$ in the group $G$ :

$$
U_{k-1}=\left\{x=(\underbrace{0, \ldots, 0}_{k-1}, x_{k}, \ldots)\right\}, \quad k=1,2, \ldots .
$$

It is easy to see that the sets $U_{k-1}$ in (8) are subgroups of $G$, and

$$
G=U_{0} \supset U_{1} \supset \ldots, \bigcap_{k=1}^{\infty} U_{k-1}=\{O\} .
$$

If we define a measure $\mu$ so that

$$
\mu\left(U_{k-1}\right)=2^{-(k-1)}, \quad k=1,2, \ldots,
$$

then in a standard way, the measure $\mu$ becomes normalized invariant with respect to the group operator "i+", the Haar-Lebesgue measure on $G$ (see, for instance [6] or [7]). A Lebesgue-Haar integral of the functions $f: G \rightarrow \mathbb{R}$ (or $\mathbb{C}$ ) with respect to the measure $\mu$ arises naturally,

$$
\int_{G} f(x) d \mu
$$

as well as the spaces $L_{p}(G), 1 \leqslant p \leqslant \infty$.

A system of Pontryagin characters of the group $G$ turns out to be Walsh-Paley system $W=\left\{w_{n}(x) ; x \in G, n=0,1, \ldots\right\}\left(w_{n}(x \dot{+} y)=w_{n}(x) \cdot w_{n}(y)\right)$. The smoothness of a function $f(x) \in L_{p}(G)$ is determined by the continuity modulus

$$
\omega_{p}(f)=\left\{\omega_{n}^{(p)}(f)=\sup _{h \in U_{n}}\left(\int_{G}|f(x \dot{+} y)|^{p} d \mu(x)\right)^{1 / p}, n=0,1, \ldots ; 1 \leqslant p \leqslant \infty\right\} .
$$


It was shown in [8] and [9] that for each sequence the identity

$$
\omega_{0} \geqslant \omega_{1} \geqslant \ldots, \quad \lim _{n \rightarrow \infty} \omega_{n}=0
$$

holds and for each $p \in[1 ; \infty]$ there exists a function $f(x) \in L_{p}(G)$ such that

$$
\omega_{n}^{(p)}(f)=\omega_{n}, \quad n=0,1, \ldots
$$

For $G$, as an analogue of the function $\frac{1}{x}$ on $(0 ; \pi)$, we can take the function

$$
K(x)=2^{k}, \quad x \in U_{k-1} \backslash U_{k}, k=1,2, \ldots .
$$

It is obvious that the function $F(x)$ in $(6)$ can be written as

$$
F(x)=\lim _{\varepsilon \rightarrow+0} \int_{\varepsilon \leqslant|t| \leqslant \pi} \frac{f(x+t)}{t} d t=\lim _{\varepsilon \rightarrow+0} \int_{\varepsilon \leqslant|t| \leqslant \pi} \frac{f(x+t)-f(x)}{t} d t .
$$

Therefore, it is natural to consider the operator

$$
(K f)(x)=-\lim _{m \rightarrow \infty} \int_{G \backslash U_{m}}(f(x+t)-f(x)) K(t) d \mu(t),
$$

for $f: G \rightarrow \mathbb{R}$, where $K(t)$ is determined by (14), and the minus sign is similar to (1), (2)). And it is natural to pose the question on relation $\omega_{p}(f)$ and $\omega_{p}(K f)$ : whether theorems by Privalov in [3] and by Bari-Stechkin in [5] hold?

The operator $K f$ in (16) was considered by the author in [10], [11]. It was established in [10] that if

$$
f(x) \sim c_{0}+\sum_{n \geqslant 0} \sum_{2^{n} \leqslant k \leqslant 2^{n+1}-1} c_{k} w_{k}(x), \quad c_{k}=c_{k}(f)=\int_{G} f(x) w_{k}(x) d \mu(x),
$$

then

$$
(K f)(x) \sim \sum_{n \geqslant 0}(n+2) \sum_{2^{n} \leqslant k \leqslant 2^{n+1}-1} c_{k} w_{k}(x) .
$$

Let

$$
g(x)=A_{0}+\sum_{n \geqslant 0} A_{n} \sum_{2^{n} \leqslant k \leqslant 2^{n+1}-1} w_{k}(x) .
$$

It was shown in [12, Lm. 3] that

$$
\omega_{n}^{(p)}(g)= \begin{cases}\sup _{k \geqslant n}\left\{2 \sum_{s \geqslant k+1}\left|\sum_{k+1 \leqslant \nu \leqslant s} 2^{\nu}\left(A_{\nu-1}-A_{\nu}\right)\right|^{p} \cdot 2^{-(s+1)}\right\}^{1 / p} & \text { as } 1 \leqslant p<\infty \\ \sup _{n \leqslant k \leqslant l}\left|\sum_{k+1 \leqslant \nu \leqslant l} 2^{\nu}\left(A_{\nu-1}-A_{\nu}\right)\right| & \text { as } p=\infty .\end{cases}
$$

Let $p=\infty$ and $A_{\nu} \searrow 0$. Then

$$
\omega_{n}^{(\infty)}(g)=\sum_{\nu \geqslant n+1} 2^{\nu}\left(A_{\nu-1}-A_{\nu}\right)
$$

and

$$
\omega_{n}^{(\infty)}(g)-\omega_{n+1}^{(\infty)}(g)=2^{n+1}\left(A_{n}-A_{n-1}\right) .
$$

This yields

$$
A_{n}=\sum_{k \geqslant n} 2^{-(k+1)}\left(\omega_{k}^{(\infty)}(g)-\omega_{k+1}^{(\infty)}(g)\right)
$$


Thus, by [11], (18),

$$
\omega_{n}^{(\infty)}(K g)=\sum_{\nu \geqslant n+1} 2^{\nu}\left(A_{\nu-1} \log (\nu-1)-A_{\nu} \log \nu\right)
$$

Relations (21) show that there exist functions in $L_{\infty}(g)$, for which theorems by Privalov and Bari-Stechkin do not hold in the space $L_{\infty}(g)$.

Let us consider another limiting case, $p=1$. Then as $A_{\nu} \searrow 0$,

$$
\omega_{n}^{(1)}(g)=A_{n}
$$

(we necessarily have $\left.A_{n}=o\left((\log n)^{-1}\right)\right)$ and

$$
\omega_{n}^{(1)}(K g)=A_{n} \log n .
$$

Hence, theorems by Privalov and Bari-Stechkin do not hold for $L_{1}(G)$.

Let us consider the case $p=2$. It was shown in [13] that for each function $f(x) \in L_{2}(G)$ possessing the Fourier-Walsh-Paley series (17) we have

$$
\left(\sum_{k \geqslant 2^{n}}\left|c_{k}(f)\right|^{2}\right)^{1 / 2} \leqslant \frac{1}{\sqrt{2}} \omega_{n}^{(2)}(f),
$$

and there exists a function $g(x) \in L_{2}(G)$, for which

$$
\left(\sum_{k \geqslant 2^{n}}\left|c_{k}(g)\right|^{2}\right)^{1 / 2}=\frac{1}{\sqrt{2}} \omega_{n}^{(2)}(g) .
$$

Earlier for the trigonometric case these facts were established by N.I. Chernych in [14].

It follows from $(22)$ and $(18)$ that theorems by Privalov and Bari-Stechkin do not hold for $L_{2}(G)$. If the sequence $\left\{A_{\nu}\right\}$ tends to zero quite fast for each $p \in[1 ; \infty]$, by $(20)$ we can obtain that theorems by Privalov and Bari-Stechkin do not hold in $L_{p}(G)$.

The results of this paper were partially announced in [15].

\section{BIBLIOGRAPHY}

1. N.K. Bari. Trigonometric series. GIFML, Moscow (1961). (in Russian).

2. N.N. Luzin. Integral and trigonometric series. GIFML, Moscow (1951). (in Russian).

3. I.I. Privalov. Cauchy integral. Sovgrafija 13 otd., Saratov (1919). (in Russian).

4. N.K. Bari. On best approximation of two conjugate functions by trigonometric polynomials // Izv. Akad. Nauk SSSR Ser. Matem. 19:5, 285-302 (1955). (in Russian).

5. N.K. Bari, S.B. Stechkin. Best approximations and differential properties of two conjugate functions // Tr. Mosk. Mat. Obs. 5, 483-522 (1956). (in Russian).

6. G.N. Agaev, N.Ya. Vilenkin, G.M. Dzhafarli, A.I. Rubinstein. Multiplicative systems of functions and harmonic analysis on zero-dimensional groups. ELM, Baku (1981). (in Russian).

7. B.I. Golubov, A.V. Efimov, V.A. Skvortsov. Walsh series and transforms. Nauka, Moscow (1981). (in Russian).

8. A.I. Rubinstein. Moduli of continuity of functions, defined on a zero-dimensional group // Matem. Zamet. 23:3, 379-388 (1987). [Math. Notes, 23:3, 205-211 (1978).]

9. S. Fridli. On modulus of continuity with respect to functions defined on Vilenkin groups // Acta Math. Hungar. 45:3-4, 393-396 (1985).

10. A.I. Rubinstein. About functions on the dyadic group and Walsh series // Anal. Math. 41:1-2, 73-81 (2015).

11. A.I. Rubinstein. On a certain integral operator acting on functions defined on the dyadic group // Eurasian Math. J. 7:1, 68-73 (2016). 
12. M.F. Timan, A.I. Rubinshtein. Imbedding of classes of functions that are defined on zerodimensional groups // Izv. VUZov. Matem. 8, 66-76 (1980). [Soviet Math. (Iz. VUZ), 24:8, 74-85 (1980).]

13. N.Ya. Vilenkin, A.I. Rubinshtein. A certain theorem of S.B. Stečkin on absolute convergence, and series in systems of characters of zero-dimensional abelian groups // Izv. VUZov. Matem. 9, 3-9 (1975). [Soviet Math. (Iz. VUZ), 19:9, 1-7 (1975).]

14. N.I. Chernykh. The approximation of functions by polynomials with constraints // Trudy MIAN SSSR. 88, 75-130 (1967). [Proc. Steklov Inst. Math. 88, 79-138 (1967).]

15. A.I. Rubinstein On the analog of the Bary-Stechkin theorem of the conjugate functions for the dyadic group // in Book of abstracts of Sixth international conference "The problems of Mathematical Physics and Mathematical modelling", Moscow, 210-211 (2017).

Alexander Iosifovich Rubinshtein,

National Research Nuclear

University MEPhI,

Kashirskoe road, 31,

115409, Moscow, Russia

E-mail: rubinshtein_aleksandr@mail.ru 